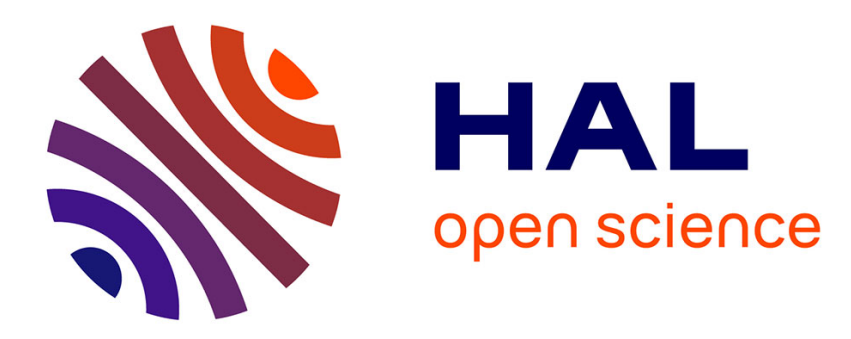

\title{
On unidirectional flight of a free flapping wing
}

Nicolas Vandenberghe, Stephen Childress, Jun Zhang

\section{To cite this version:}

Nicolas Vandenberghe, Stephen Childress, Jun Zhang. On unidirectional flight of a free flapping wing. Physics of Fluids, 2006, 18, pp.014102. 10.1063/1.2148989 . hal-00018862

\section{HAL Id: hal-00018862 https://hal.science/hal-00018862}

Submitted on 7 Sep 2015

HAL is a multi-disciplinary open access archive for the deposit and dissemination of scientific research documents, whether they are published or not. The documents may come from teaching and research institutions in France or abroad, or from public or private research centers.
L'archive ouverte pluridisciplinaire $\mathbf{H A L}$, est destinée au dépôt et à la diffusion de documents scientifiques de niveau recherche, publiés ou non, émanant des établissements d'enseignement et de recherche français ou étrangers, des laboratoires publics ou privés. 


\title{
On unidirectional flight of a free flapping wing
}

\author{
Nicolas Vandenberghe \\ Applied Mathematics Laboratory, Courant Institute, New York University, New York, New York 10012 \\ and IRPHE, Université de Provence, 13384 Marseille, France \\ Stephen Childress \\ Applied Mathematics Laboratory, Courant Institute, New York University, New York, New York 10012 \\ Jun Zhang ${ }^{\text {a) }}$ \\ Department of Physics, New York University, New York, New York 10003 \\ and Applied Mathematics Laboratory, Courant Institute, New York University, New York, New York 10012
}

(Received 3 August 2005; accepted 8 November 2005; published online 6 January 2006)

\begin{abstract}
We study the dynamics of a rigid, symmetric wing that is flapped vertically in a fluid. The motion of the wing in the horizontal direction is not constrained. Above a critical flapping frequency, forward flight arises as the wing accelerates to a terminal state of constant speed. We describe a number of measurements which supplement our previous work. These include (a) a study of the initial transition to forward flight near the onset of the instability, (b) the separate effects of flapping amplitude and frequency, (c) the effect of wing thickness, (d) the effect of asymmetry of the wing planform, and (e) the response of the wing to an added resistance. Our results emphasize the robustness of the mechanisms determining the forward-flight speed as observed in our previous study. () 2006 American Institute of Physics. [DOI: 10.1063/1.2148989]
\end{abstract}

\section{INTRODUCTION}

The flapping of wings, fins, and other appendages is a common mode of locomotion in nature. ${ }^{1}$ As a wing or fin is flapped in one direction, thrust can be generated in the perpendicular direction. ${ }^{2}$ This thrust will balance resistance as the animal reaches a state of steady flight or swimming. Thrust generation from a flapping wing was first discussed by Knoller ${ }^{3}$ and Betz, ${ }^{4}$ and has been quantitatively studied in the framework of inviscid flow theory. ${ }^{2,5}$ Previous experiments ${ }^{6-8}$ have focused on the efficiency of the flapping mechanism for underwater locomotion. In these experiments, a wing performs heaving and/or pitching motion with an imposed forward speed. The generated thrust is shown to depend strongly on the Strouhal number $\mathrm{St}=f a / U$, where $f$ is the flapping frequency, $a$ the peak-to-peak flapping amplitude, and $U$ the flow speed. It was found from previous experiments that the generated thrust is an increasing function of the Strouhal number, at least in the range of $0.2<\mathrm{St}$ $<0.5$. Optimal power generation (the ratio between the generated power and the power needed to drive the prescribed flapping motion) is reached when St is between 0.3 and 0.4 (Refs. 8 and 9). In this range of Strouhal number, the wake structure associated with the forward flight takes the form of an inverted von Kármán vortex street, a flow pattern also observed in the wake of a swimming fish. ${ }^{10,11}$

In a recent study, ${ }^{12}$ we examined the question of how flapping flight might emerge through the interaction of a simple body with its surrounding fluid. Our experimental approach is different from the classical one. Apart from the prescribed flapping motion, we neither initiate nor sustain the forward motion of the wing. Instead, the wing is allowed to

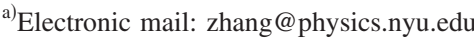

select its own speed as it seeks to balance hydrodynamical and external forces. Here, the wing is driven by a prescribed vertical flapping motion but is free to move horizontally. At low flapping frequency, because of the dominance of fluid drag, we observe a regime with no horizontal motion of the wing. Above a critical flapping frequency, however, the wing spontaneously starts to move horizontally, and accelerates until a terminal speed is reached. The wing is fore-aft symmetric and forward flight emerges as a result of spontaneous symmetry breaking, when the Reynolds number associated with the flapping frequency exceeds a threshold. ${ }^{12}$ In particular, the critical Reynolds number was found to be approximately 10 .

The results of Ref. 12 confirm, for simple reciprocal flapping of a wing with fore-aft symmetry, the bifurcation that was theorized in Ref. 13. In that paper, the conjecture of a critical Reynolds number was based on observations of the swimming of the pteropod mollusk (Clione antarctica). Those observations showed that their wings became ineffective at a Reynolds number (based upon flapping frequency and body length) below 10. Related arguments in favor of a transitional, intermediate Reynolds number range for natural flapping flight were given in Ref. 14.

A consequent numerical study by Alben and Shelley ${ }^{15}$ on the dynamics of a two-dimensional (2D) flapping body in a 2D viscous fluid reproduced many of the qualitative aspects of our experiment. They showed that unidirectional locomotion resulted from an instability related to that of von Kármán for symmetric wakes behind bluff bodies. Typically, this instability becomes operative for a frequency-based Reynolds number on the order of 10 . They also studied the wing shape and inertia, factors affecting the performance of forward flight.

In the work reported here, we study this basic phenom- 

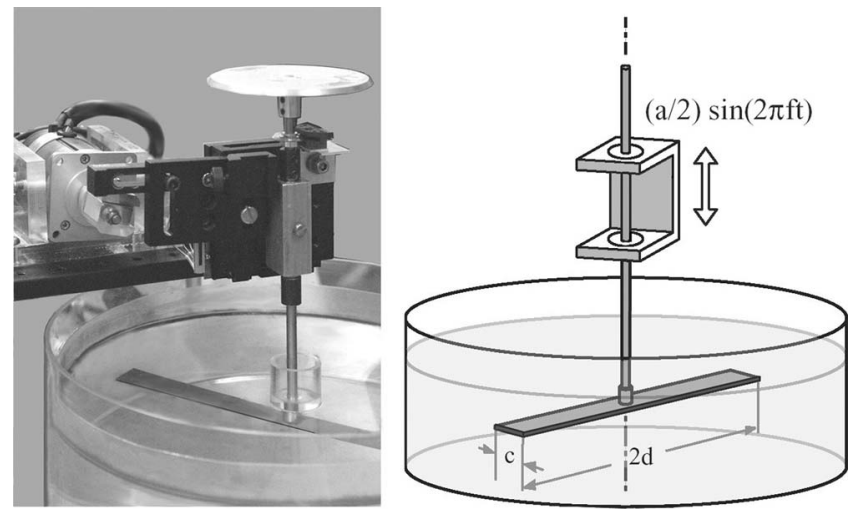

FIG. 1. The experimental setup. A rigid, rectangular wing is flapped up and down sinusoidally. It is allowed to rotate freely in the horizontal plane. The total length of the wing $(2 d)$ is $25.7 \mathrm{~cm}$, its width (chord, c) $1.9 \mathrm{~cm}$, and its thickness $0.16 \mathrm{~cm}$. Both flapping amplitude and frequency can be adjusted to study the response of flapping motion of the wing and its surrounding fluid (water). A photograph taken from the setup is shown on the left and the schematic is shown on the right.

enon in more detail and consider elaborations of the experiment. We show the stochastic nature of the onset time to locomotion, and examine the separate effects of flapping amplitude and frequency. We are particularly interested in the robustness of flapping flight. To study this, we examine factors that influence performance, such as the wing thickness and flexibility, and the effect of asymmetry of the wing. Practically, those factors are of great importance when flapping locomotion is adapted in the biological world. Finally, we study the effect of an external damping on the system, showing again the robustness of locomotion to this external factor.

\section{EXPERIMENTAL SETUP}

The experiment is conducted in a rotational geometry, shown in Fig. 1. A rigid wing with rectangular cross section is mounted at the center to a rigid shaft. A serving mechanism translates a rotational motion from a motor to a linear sinusoidal motion (shown in the photo on the left). The shaft is driven in the vertical direction through two low-friction ball bearings. The shaft together with the wing is able to rotate in the horizontal direction. Only the vertical motion of the wing is prescribed. Thus, the rotation of the wing about the vertical axis is neither imposed nor constrained. If there is any wing rotation about the shaft, in either direction, it depends entirely on the interaction between the flapped wing and the surrounding fluid. If no vertical driving is imposed, any rotation of the wing is damped by viscous forces in the fluid and the wing eventually stops. There is also, of course, some friction in the ball bearings, ${ }^{12}$ opposing the tendency to possible horizontal motion.

The advantage of using a rotational geometry is that the linear distance and thus the time needed for the system to reach a steady motion is unlimited. As we shall show, it is also relatively easy to apply precise resistance to a rotating wing in order to study the amplitude of the generated thrust. Shortcomings of the rotational geometry include the lack of precise definition of a linear speed and possible three- dimensional effects, which differ from the case of rectilinear translation. However, flow visualization reveals that the flow structure is essentially two-dimensional due to the large aspect ratio of the wing. ${ }^{12}$

The wing used in the experiment is made of a $0.16 \mathrm{~cm}$ thick $(\delta)$ stainless steel plate. It is $1.9 \mathrm{~cm}$ wide (the chord length, $c$ ) and $25.7 \mathrm{~cm}$ long $(2 d)$. The flapping motion of the wing has the form $h(t)=(a / 2) \sin (2 \pi f t)$, where $a$ is the peakto-peak flapping amplitude and $f$ the frequency of flapping (Fig. 1). The quantity af gives a measure of the flapping speed. Throughout the experiment, we use water as the working fluid. The depth of water is $15 \mathrm{~cm}$, confined in a cylindrical tank. The fluid tank is covered with a rigid lid that allows the driving shaft to pass through in the middle, so that the system has similar boundary conditions at both the top and bottom.

The Reynolds number for the vertical driving is defined through the flapping frequency $f$ and amplitude $a$, as

$$
\operatorname{Re}_{f}=a f c / \nu,
$$

where $\nu$ is the kinematic viscosity of water. As reported in our earlier work, ${ }^{12}$ above the critical threshold the flapping wing spontaneously sets out in horizontal motion, and the resulting rotation of the wing may be referred to as the "forward flight."

\section{EXPERIMENTS AND OBSERVATIONS}

\section{A. The general behavior of the forward flight}

As we observed previously, the flapping wing shows no sign of rotation when the driving Reynolds number $\operatorname{Re}_{f}$ is sufficiently small. This regime of $\mathrm{Re}_{f}$ is at the lower end of the "intermediate" range of the Reynolds number, wherein neither inertial nor viscous forces are dominant. It lies above and is an extension of the "Stokesian realm," occurring when $\operatorname{Re}_{f}$ is so small that Stokes' equations offer an accurate description of the fluid dynamics. Within the Stokesian realm it is known that forward flight of an oscillating wing will not occur, ${ }^{16,17}$ and our observations show that this situation extends out to a finite value of $\operatorname{Re}_{f}$ within the intermediate regime.

Above a threshold, either by increasing the flapping frequency or the flapping amplitude, a spontaneous symmetry breaking bifurcation takes place as the wing starts to rotate in either direction. We review now our observations of this spontaneous forward flight.

\section{Near the onset: Bifurcation from localized flapping to forward flight}

Figure 2 shows the relationship between the flapping frequency and the resulting rotational speed. Both numbers are shown in the form of the driving Reynolds number $\operatorname{Re}_{f}$ and the rotational Reynolds number $\operatorname{Re}_{\Omega}$. Here, $\operatorname{Re}_{\Omega}=c D \Omega / \nu$, where $\Omega$ is the angular speed and $D \Omega$ is the representative linear speed of the rotating wing. Distance $D=3 d / 4$ is the representative length of the wing, and the coefficient of $3 / 4$ is obtained by comparing the inertial torque with the viscous torque. $^{12}$ 


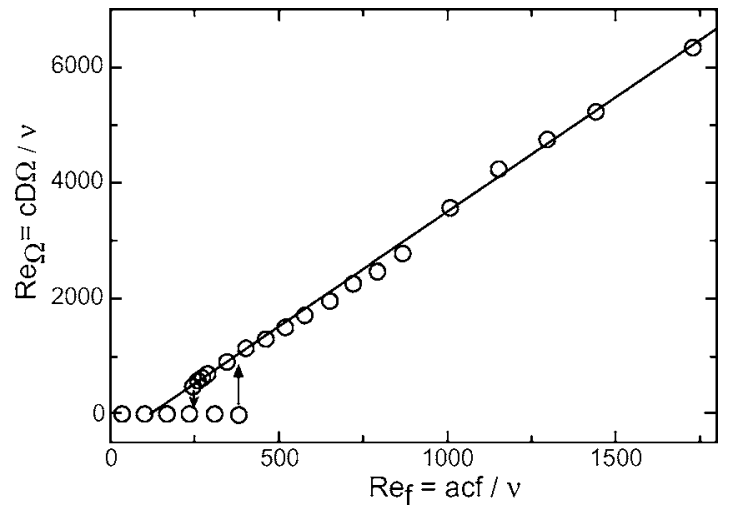

FIG. 2. Relationship between flapping frequency and rotational speed. When the flapping amplitude is fixed at $15.7 \mathrm{~mm}$, the resulting forwardflight speed $D \Omega$ is a linear function of the flapping frequency. Hysteresis is seen near the onset, likely due to the finite friction at the shaft.

Figure 3 shows a typical flow field using micrometersized hydrogen bubbles that suspend in water. A thin, vertical light sheet is introduced into the fluid tank and intersects the passing-by wing. Digital photographs with 1/20th of second exposure render flow streak lines around the flapping wing. Within the highly structured wake, all eddies with alternating signs pair up with their neighbors to form an "inverted" von Kármán vortex street. ${ }^{10,11}$ The flow structure becomes more complicated near wing tips and the shaft. Its influence to the flapping wing is arguably negligible due to its limited spatial presence. In a broad neighborhood of $D$ (between $0.5 d$ and $0.9 d$ ), however, the vortical structure left by the wing show qualitatively the same morphology, indicating a quasi-2D flow field. The streak lines of the hydrogen bubbles (Fig. 3, top) offer an indication of the contrast between the fluid velocity before the wing (on the far right edge of the photograph) and that after the passage of the wing. The residual wake velocity perturbations are estimated to be less than $7 \%$ of the velocity immediately after the passage of the wing.

The rotational speed depends linearly on the flapping frequency. Well above the threshold, once the flapping fre-

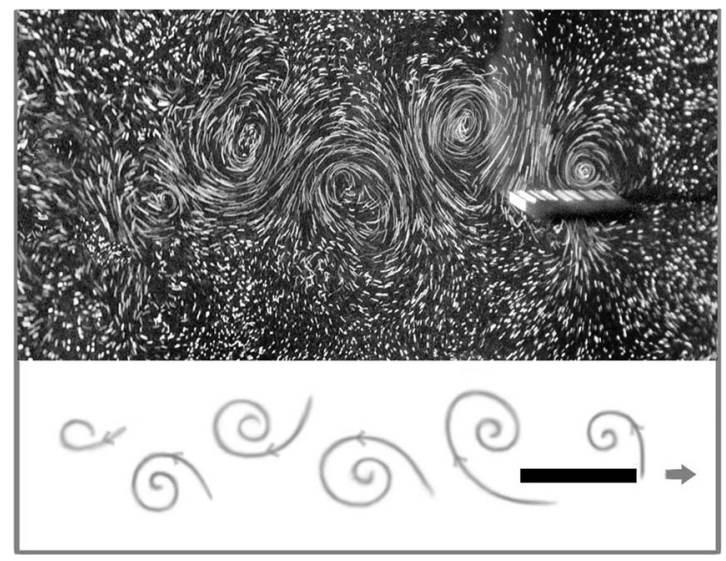

FIG. 3. Flow visualization around the flapping wing. A vertical light sheet is introduced into the fluid tank at position near $D$. The wake of the flapping wing that is in forward motion exhibits an "inverted" von Kármán vortex street. The corresponding illustration of the flow directions is sketched at the bottom.

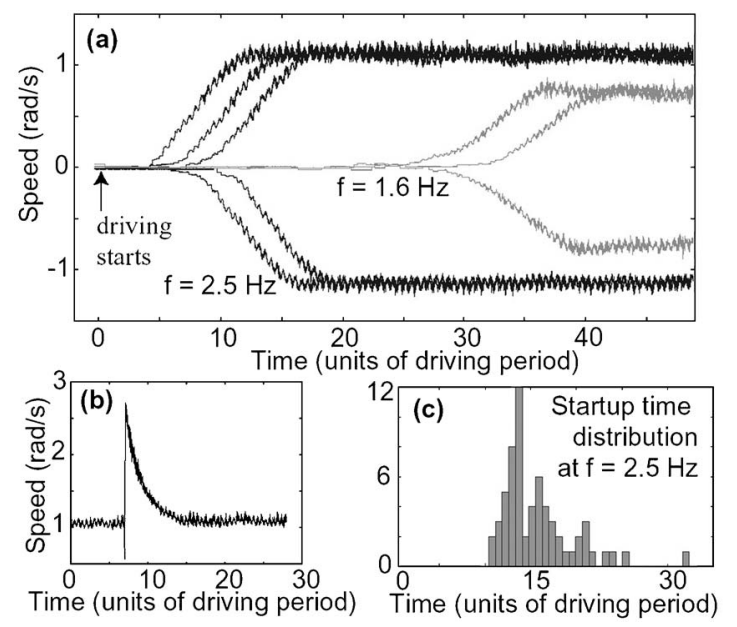

FIG. 4. Transition to rotation at a fixed flapping amplitude $a=15.7 \mathrm{~mm}$. (a) Above the onset, as the wing starts to flap, the time to reach the terminal flight speed shortens as the flapping frequency is increased. (b) One typical time series shows how a flapping wing that is pushed externally to a higher speed decays monotonically to its terminal speed. Both measurements show the uniqueness of the terminal speed at a fixed driving Reynolds number. (c) At fixed Reynolds number (when $a=1.57 \mathrm{~cm}$ and $f=2.5 \mathrm{~Hz}$ ), a distribution of times for the wing to reach $85 \%$ of the terminal speed.

quency is fixed, the steady forward-flight speed is uniquely determined (modulo the clockwise and counterclockwise symmetry). Several time series in Fig. 4(a) show how the speed is reached after the wing starts to flap at time zero. It takes many flapping cycles to reach the steady flight. In particular for data taken close to onset $(f=1.6 \mathrm{~Hz})$ the wing spends a long time wandering about the stationary position before it settles into forward flight, a dynamic state with an apparent basin of attraction. The rotational speed grows smoothly before reaching the terminal speed. Well above the onset, at $f=2.5 \mathrm{~Hz}$, the lead time to reach a steady forward motion is greatly reduced. The small-scale oscillations apparent in Fig. 4(a) correspond to the variations of the rotational speed during one driving cycle.

We observe that the wing rotates in either direction with essentially equal probability. In one experiment, among 61 trials, 29 led to rotation in one direction, 32 in the other. Between adjacent trials, we let the system relax for at least 3 min, without the wing flapping and without any external disturbance. Figure 4(c) shows the distribution of time the system takes to reach a speed that is within $15 \%$ of its final speed.

\section{Well above the onset: Uniqueness of the forward flight state}

Multistable states are frequently observed when a moving object interacts with a flow at high Reynolds number. Typical examples include coexisting patterns in a threedimensional cylinder wake ${ }^{18}$ or the fluttering of an elastic structure in a parallel flow. ${ }^{19,20}$ In our experiment, possible multistable states could result in the nonuniqueness of the rotational speed. That is, for a given flapping Reynolds number $\mathrm{Re}_{f}$, different rotational Reynolds numbers $\mathrm{Re}_{\Omega}$ could be observed, depending on the initial conditions. To check this point, we start a rotational motion by hand while the wing is 
flapped at a fixed frequency. As we show in Fig. 4(b), when the wing is launched well above the steady forward-flight speed $\Omega$, and then left in unperturbed flapping motion, it quickly relaxes to $\Omega$ within just a few flapping cycles. We performed several similar trials and found that, well above the onset of forward flight, no other stable solution is found for the same Reynolds number $\operatorname{Re}_{f}$. The forward-flight speed is uniquely selected by the driving parameters, modulo the clockwise and counterclockwise symmetry.

Close to the onset of the forward-flight motion, however, we observe a hysteretic behavior or bistability between the nonrotating and rotating states. This behavior was reported and discussed in our previous paper. ${ }^{12}$ It is likely due to the finite friction of the ball bearings present in our experiment. Recent numerical simulations suggest that, under different conditions, the bifurcation to forward flight of a vertically flapping wing may be different from what is depicted here. In a frictionless situation, more complex transition scenarios were found, sometimes involving intermediate chaotic states. $^{15}$

Moreover, we also tested the effect of the fluid tank geometry: different tanks with either circular base or square base of different sizes do not seem to yield qualitative or quantitative difference (say, for example, with different tanks, data sets obtained on rotational speed versus driving frequency collapse onto each other well within the measurement error bars). When the fluid depth is sufficiently shallow, however, the dynamics would definitely be affected. This boundary influence is connected with the so-called "ground effect" of flying birds. This is an issue that will be discussed elsewhere.

\section{B. The influence of different parameters on a rigid wing}

In this section, we discuss factors that directly affect the performance of the forward flight. Those factors are the flapping amplitude, and the thickness of the wing. During the flapping motion, the wing remains rigid with no apparent deflection in each case.

\section{The effect of the flapping amplitude}

In the expression of the flapping Reynolds number $\mathrm{Re}_{f}$, the flapping amplitude $a$, and the flapping frequency $f$ influence the dynamics as the product $a f$. Amplitude is expected to play a role similar to that of frequency. This conjecture needs to be tested, since the ratio $a / c$ of amplitude to chord length is an independent parameter of possible importance. At fixed flapping frequency, the flapping amplitude is adjusted in small steps. We test flapping amplitudes in a range between 0.4 and 1.3 times $c$. We then measure the resultant flight speed. Figure 5 shows how rotational speed changes as a function of flapping amplitude.

It can be argued that the amplitude of the flapping motion should be limited within a finite range. Compared to the chord length of the wing, if the amplitude is too small, the wing appears to be a large extended plate; the size of the vortices generated on both edges, which are crucial for wake formation and thrust generation, would be relatively smaller.

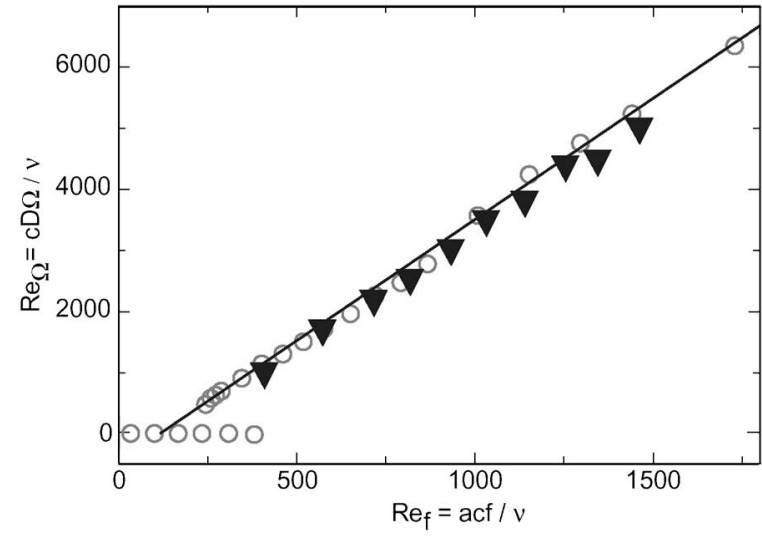

FIG. 5. The effect of flapping amplitude (shown as $\mathrm{Re}_{f}$ ) on rotational speed $\left(\operatorname{Re}_{\Omega}\right)$ : At a fixed flapping frequency, $f=2.5 \mathrm{~Hz}$, the flapping amplitude is adjusted incrementally to change the driving Reynolds number $\operatorname{Re}_{f}$. The resulting terminal speed of the forward-flight wing (solid triangles) follow closely the data obtained as one changes the frequency only (circles, same data shown in Fig. 2). The amplitude changes from 0.73 to $2.54 \mathrm{~cm}$, which is 0.38 to 1.32 chord length.

If the flapping amplitude is far above the chord length, however, energy dissipation will primarily take place in the vertical direction. In both situations, either with small or large amplitudes, the system would not favor energy transfer to the lateral direction, thus preventing effective forward flight. Apparently, the range of the flapping amplitude (0.4 to 1.3 chord length) guarantees that the generation of lateral thrust maintains the linear relationship.

In addition to being a dimensionless number used to characterize wake dynamics behind an obstacle in a moving fluid, ${ }^{21}$ the Strouhal number is also used to characterize animal locomotion such as swimming of fish ${ }^{22}$ and flying of birds. ${ }^{23}$ For effective thrust generation from flapping, the Strouhal number usually falls within a small neighborhood of 0.30 (Ref. 8). It should be noted that for a fixed bluff body such as a circular cylinder, the corresponding Strouhal number is about $30 \%$ smaller, St $\sim 0.20$. The flow structures behind the resistive cylinder and a swimming fish are different, on the one hand a typical von Kármán vortex street, and on the other an inverted von Kármán wake., ${ }^{2,10,11}$ This has been observed in previous flapping-wing experiments. ${ }^{24}$ In our study, the Strouhal number is the ratio between $\operatorname{Re}_{f}$ and $\operatorname{Re}_{\Omega}$ : $\mathrm{St}=\mathrm{Re}_{f} / \mathrm{Re}_{\Omega}$. Since we observe an offset for $\mathrm{Re}_{\Omega}$ (Figs. 2 and 5), the Strouhal number decreases with increasing $\operatorname{Re}_{f}$. However for large values of $\mathrm{Re}_{f}$, the Strouhal number converges to a constant value, which is the inverse slope of the straight line shown in Figs. 2 and 5. The Strouhal numbers obtained from both Figs. 2 and 5 are between 0.26 and 0.28 . It is clear that the effects of amplitude and frequency of the flapping on the dynamics of the flapping wing is determined by their product. To realize a desired forward-flight speed, one can vary either one of the variables.

\section{The effect of the wing thickness}

Changing the wing thickness $(\delta)$ has several effects. For a nonflapping wing, increasing the thickness increases the form drag, and thus the damping of the horizontal motion is 


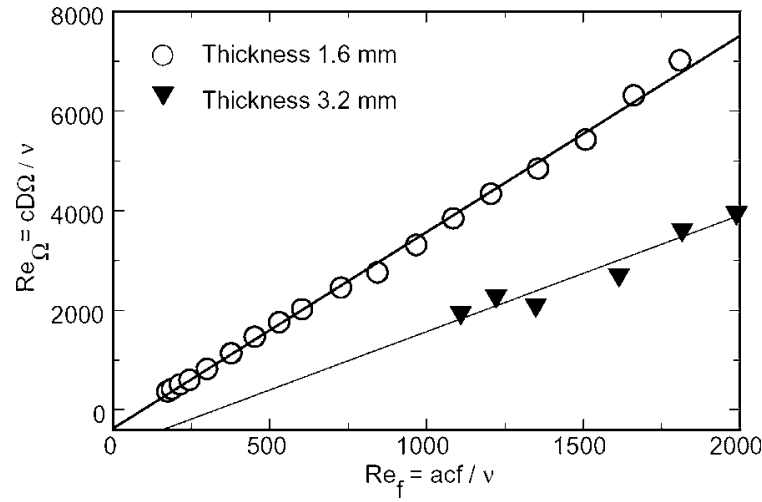

FIG. 6. Effect of the thickness of the wing. As the thickness is doubled, the rotational speed decreases. The inverse slope (the Strouhal number) of the fitted straight lines is about 0.26 for a wing with thickness of $1.6 \mathrm{~mm}$ and 0.43 for thickness of $3.2 \mathrm{~mm}$.

stronger. The thickness ratio $\delta / c$ modifies the geometry at the edge of the wing and thus modifies vortex generation. For example, it has been observed that the lift of a 2D flapping elliptic airfoil decreases as the thickness ratio increases (i.e., when the wing becomes thicker). ${ }^{25}$ Thus, in our experiment, we expect to see reduced horizontal speed as the thickness increases because of both of these effects.

From Fig. 6, we see that if the thickness is doubled, from 1.6 to $3.2 \mathrm{~mm}$ ( $8.4 \%$ to $16.8 \%$ of the chord), the ratio between the flapping Reynolds number and the resulting rotational Reynolds number-the Strouhal number-increases from 0.26 to 0.43 , showing that forward-flight speed deceases as the wing thickness is increased.

As we increase the wing thickness even further, the two effects mentioned above become more pronounced. For a thickness of $\delta=4.8 \mathrm{~mm}$, we do not observe forward flight. In this case, the thick wing only performs the prescribed vertical flapping motion but with very small horizontal excursions, much less than the chord length. The resistance of the thick wing prevents the transition to forward flight.

\section{The effects of flexibility}

In the biological world, bird wings and fish fins are built from supporting bones and soft tissues. They are actively driven by groups of muscles in order to achieve a prescribed motion. Due to their flexibility, however, fluid forces and the inertia of the wing may either reduce or exaggerate the intended flapping motion and thereby affect performance in forward flight.

So far, we have explored the effects of parameters such as wing thickness, flapping frequency, and amplitude. In these experiments, the wing remains rigid. The fluid forces are not strong enough to produce significant deformation to the wing. To study the dynamics of a flexible wing within our current setup, we use a flexible, plastic (acrylonitrile butadiene styrene) wing to replace the original rigid one (stainless steel). It has the same thickness $(\delta=0.16 \mathrm{~cm})$, chord $(c=1.9 \mathrm{~cm})$, and length $(2 d=25.7 \mathrm{~cm})$, but the Young's modulus $E$ is much lower than that of stainless steels, around $1.9 \mathrm{GPa}$. The fluid force due to flapping is now strong enough to bend the wing. We estimate that the pres-

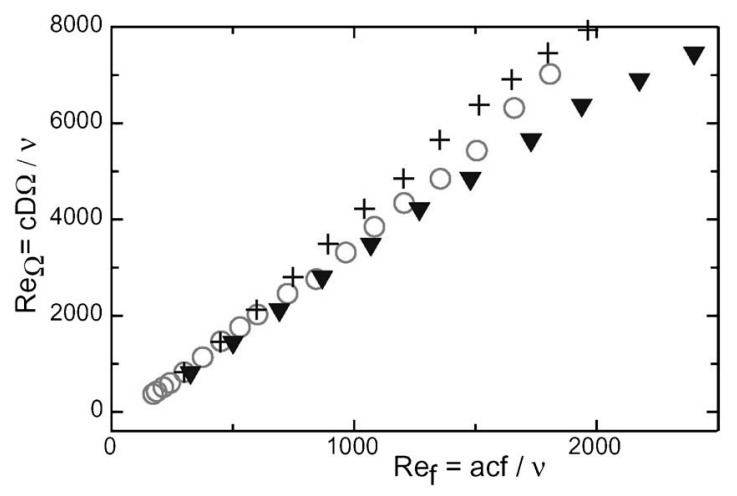

FIG. 7. The effect of wing flexibility (crosses and triangles). A plastic wing of the same size as the one shown in Fig. 1, rotates faster than the rigid wing (circles, data from Fig. 2). The flapping amplitude used to calculate $\operatorname{Re}_{f}$ is the amplitude at the shaft (crosses, $a=1.57 \mathrm{~cm}$ ) or the measured amplitude at the wing tips (triangles). Due to the flexibility of the plastic wing, the actual flapping amplitude is greater than the intended one near the wing tips, yielding faster angular speeds.

sure that acts on the flapping wing is on the order of $p=C_{D} \rho(\pi a f)^{2} / 2$ at large Reynolds numbers, where $\pi a f$ is the maximal speed of the wing in the vertical direction. The drag coefficient $C_{D}$ is here taken to be 1 . At $f=6 \mathrm{~Hz}$, $a=1.57 \mathrm{~cm}$, the fluid pressure $p$ is on the order of $44 \mathrm{~Pa}$. Now, the fluid force would give rise to $12 p d^{4} / 8 E \delta^{3}$ $=2.3 \mathrm{~mm}$ of deformation. Note that dynamical effects are not included in this simple computation. In particular, the estimate of the force does not take into account the unsteady nature of the flow. The drag coefficient adopted here is a conservative estimate: for oscillating wing, $C_{D}$ could typically be of the order of 4 or 5 (Ref. 26).

During forward flight, the fluid forces acting on the flexible wing are much harder to estimate. We find that the total vertical displacement of the wing tips increased progressively with increased flapping frequency. For instance, at $f=6.0 \mathrm{~Hz}$, the wing tip amplitude is about $34 \%$ higher than the intended flapping amplitude. Figure 7 shows the response of the plastic wing to the flapping motion. There are two sets of data presented there. The crosses show the relation between flapping frequency and rotational speed, while the flapping amplitude is taken to be $1.57 \mathrm{~cm}$, or 0.83 times the chord length, which is the intended flapping amplitude. This set of data shows that the flexible wing rotates more quickly than the rigid one. The triangles in the same figure are drawn from the same measurement, but the amplitude used to compute the flapping Reynolds number $\mathrm{Re}_{f}$ is the actual amplitude measured near the wing tips at each frequency. For this flapping amplitude, the wing rotation is reduced. We believe that the discrepancy between the flexible and the solid wing would become smaller if the flapping amplitude were measured between the shaft and the wing tip.

Under the same driving, we observe that the rotational speed for a flexible wing increases relative to a rigid wing. The tip of the wing moves further due to its flexibility and the imposed fluid forces. The increased flapping amplitude yields higher speed. 


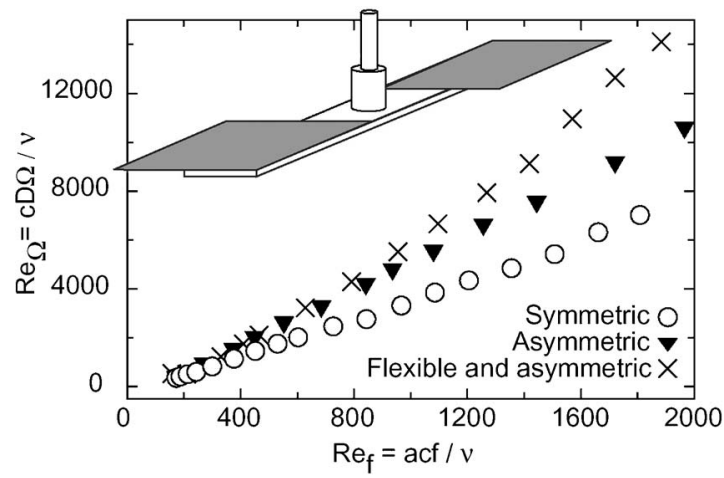

FIG. 8. Wing with broken symmetry. Extension plates are added to break the clockwise/counterclockwise symmetry. The plates were made of rigid copper (shown as triangles) or of flexible Mylar sheets (crosses). The result from the symmetric wing is added for comparison (circles, data from Fig. 2). For the two asymmetric wing, $\operatorname{Re}_{\Omega}$ was computed using the distance from the driving shaft to the middle of the wide section $(D=86 \mathrm{~mm})$. The flapping amplitude is fixed at $a=1.57 \mathrm{~cm}$.

\section{A wing with broken symmetry}

We dealt here with wings that are designed with perfect fore-aft symmetry as we perform each experiment. Robust, spontaneous symmetry breaking has been observed in all cases. It is also of interest to study the dynamics of a wing where symmetry is broken in the geometry of the planform.

\section{Broken symmetry resulted from a flexible attachment to the rigid wing}

For a symmetric wing, its direction of rotation is selected randomly by the system with equal probability. We also find that the wing performs equally well in both directions, as far as the measurements of flight velocity are concerned.

We now break the symmetry of the system. Figure 8 shows a schematic of a modified wing. On each end of the wing, a flexible extension is mounted so that the planform becomes asymmetric (see the shaded part of Fig. 8). The extension is made of $50 \mu \mathrm{m}$ thick Mylar ${ }^{\circledR}$ sheet, having a Young's modulus of $5 \mathrm{GPa}$. Each extension is $85 \mathrm{~mm}$ long and $40 \mathrm{~mm}$ wide.

We observe that forward flight starting from rest is always counterclockwise when seen from above. If the wing is forced to rotate in the opposite direction, it takes less than 10 flaps for the wing to slow down and reverse the direction. In Fig. 8, the equivalent wing span $D$ is taken from the shaft to the middle of the wide section. Its width $(40 \mathrm{~mm})$ is used as the chord to compute the flapping Reynolds number. The forward speed is significantly greater than that of the "regular" wing.

\section{Effect of a rigid wing with broken symmetry}

We next replace the flexible attachment by a rigid copper extension. It has exactly the same planform as the flexible extension, but the thickness is increased to $0.23 \mathrm{~mm}$.

Under driving, the wing quickly starts the same unidirectional motion as in the flexible case. Figure 8 also shows $\operatorname{Re}_{\Omega}$ as a function of $\operatorname{Re}_{f}$. The rigid asymmetric wing behaves essentially the same as the original wing, except that the direction is predetermined and the rotation rate is somewhat greater. For this geometry, the linear speed is also based on the distance from the shaft to the middle of the attachment. The corresponding slope between $\operatorname{Re}_{f}$ and $\operatorname{Re}_{\Omega}$ gives a Strouhal number 0.22 .

\section{E. Effect of external damping, added as friction}

As noted earlier, thrust production by generating eddies is a key element for understanding the motion of the wing. We should carefully distinguish the hydrodynamical torque that results from interaction between the wing and the surrounding fluid, and an external torque applied to the wing. The hydrodynamical torque can be positive (thrust production) or negative (drag). External torque originates from bearing friction or magnetic damping (see below). If no external torque were present, the observed rotation would be a state with no net hydrodynamical torque. In the presence of a resistive (i.e., negative) external torque, once the wing finds its terminal-state speed, the hydrodynamical torque has to be positive to balance the resistance. We discuss now how much positive hydrodynamical torque can be produced by the flapping wing.

To add controlled resistance to the shaft, a horizontal aluminum disk is attached at its top (Fig. 1, left). It rotates together with the rotating wing. A magnet is placed facing the disk at a constant distance: the disk and the magnet are driven up and down in the vertical direction but the magnet does not rotate with the disk. When the disk (with the wing) starts to rotate, a torque that opposes the rotation is produced as a result of an induced current within the conductive disk. ${ }^{27}$ This torque is proportional to the rotational speed, $T_{\text {res }}=-\nu_{m} \Omega$, where $\nu_{m}$ is a proportionality constant that can be adjusted by changing the distance between the magnet and the disk. During the experiment, we fix the driving Reynolds number $\mathrm{Re}_{f}$ by fixing both the flapping amplitude $(a=1.57 \mathrm{~cm})$ and frequency $(f=2.5 \mathrm{~Hz})$. We then change the distance between the magnet and the disk incrementally, in order to vary the proportionality constant $\nu_{m}$. For each $\nu_{m}$, the external, resistive torque is measured by recording the free decay of the wing rotating in the empty fluid tank (i.e., without hydrodynamical torque, air drag being negligible). With the wing mounted and flapped in water, we then record each terminal speed of the wing after a sufficient number of flaps. Figure 9 shows the resistive torque $T_{\text {res }}$ and its corresponding power $\Omega T_{\text {res }}$ as functions of the Strouhal number. As the proportionality constant $\nu_{m}$ increases, the wing slows down in rotation, increasing the Strouhal number. For high rotational speed (when $\mathrm{St}<0.5$ ), the torque first increases and reaches a maximum around $\mathrm{St} \sim 0.5$ as the external resistance is increased. As the external damping is further increased, the rotational speed keeps on decreasing and the resistive torque reaches a plateau at about $2.2 \times 10^{-4} \mathrm{Nm}$.

It is interesting that at low Strouhal numbers torque can be developed with little change in rotational speed, attaining at $\mathrm{St} \sim 0.5$, a state of maximal power output. Indeed, it has been shown that in the range of $0.2<\mathrm{St}<0.4$, thrust production strongly depends on the Strouhal number and is associated with the formation of an inverted von Kármán vortex 

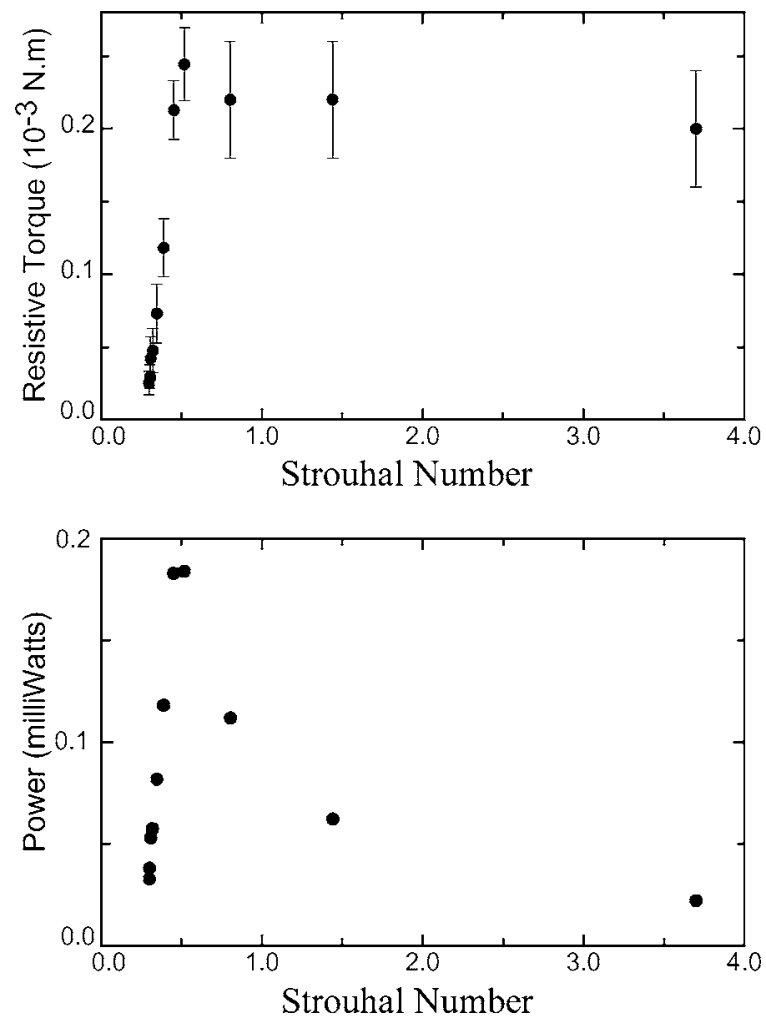

FIG. 9. In the presence of an external damping, and at fixed flapping amplitude $(a=1.57 \mathrm{~cm})$ and frequency $(f=2.5 \mathrm{~Hz})$, we study the response of the system to different external loads. We here show the torque $\left(T_{\text {res }}\right.$, top figure), and the power produced ( $T_{\text {res }} \Omega$, bottom figure), vs the Strouhal number.

street. ${ }^{8}$ On the higher end of this range, the flow structure changes, and in particular leading edge separation may be substantially stronger and thus the thrust-producing wake structure can be eroded. ${ }^{28}$ Surprisingly, we find that for higher Strouhal number $(\mathrm{St}>0.5)$ the amount of thrust produced maintains the same level until $\mathrm{St} \sim 3.7$. This range of Strouhal number has not been studied in detail in the literature, and it would be interesting to conduct further investigations, in particular to elucidate the nature of the flow structure for these high values of St. Once more, what is remarkable here is the robustness of the forward flight. With the added damping, the rotational speed adapts to maintain essentially constant torque.

\section{DISCUSSION AND CONCLUSION}

We have studied the dynamics of a wing that is flapped up and down but is free to rotate horizontally, to gain insight into the transition to forward flapping flight. The present work extends the experimental findings reported earlier. ${ }^{12} \mathrm{At}$ a critical flapping Reynolds number, the wing spontaneously starts to rotate. This transition is a result of spontaneous symmetry breaking with the two (rotation) flight directions equally probable. The rotational speed is dynamically selected through the balance of fluid and external forces. Flapping amplitude and flapping frequency play similar roles: increasing either parameter would cause the flight speed to increase linearly in the limited range considered. Overall, it appears that the mechanism selecting the rotational speed is remarkably robust: we obtained the same relation between $\mathrm{Re}_{f}$ and $\mathrm{Re}_{\Omega}$ for various flapping amplitudes and frequencies.

In this work, we further investigated the effects of the different parameters of the wing affecting the rotational speed. In particular, the wing thickness is critical to the rotation. With a thick wing, no rotation is observed. It is likely that a thick wing experiences significant form resistance at its leading edge, and is also less effective in the shedding of eddies that help the formation of a thrust-generating wake.

The elasticity of the wing also affects the dynamics of the wing. Some increase in forward speed occurs for a flexible wing with fore-aft symmetry (Fig. 7). The effect is further enhanced for an asymmetric wing, most probably the result of the allowed twisting of the flexible additions to the rigid wing (Fig. 8). In the biological world, wings and fins are indeed flexible. For the more complex motions found in nature, twisting and bending of the wing combine to modify the aerodynamic forces. Further studies are required to investigate in more detail the role of elasticity, and to elucidate the structures that are most effective for forward flight.

We have also shown that when an external force is applied, the motion adapts its Strouhal number (forward speed) to the excess drag. Slow rotation can be sustained even when the Strouhal number reaches as high as 3.7. We also determined the power that a wing can produce: it appears that the wing produces maximal power at a Strouhal number of about 0.5 .

Overall our investigation suggests that the transition to forward flapping flight is a robust phenomenon: it works for various (sufficiently thin) wing geometries, flapping frequency and amplitude. It produces roughly the same magnitude of force (or torque in the rotational geometry) even at very low speed (high Strouhal number).

\section{ACKNOWLEDGMENTS}

We wish to thank L. Rosellini and M. Shelley for fruitful discussions. This project is supported by the Department of Energy (Contract No. DE-FG0200ER25053).

${ }^{1}$ S. Vogel, Life in Moving Fluids, 2nd ed. (Princeton University Press, Princeton, 1994).

${ }^{2} \mathrm{~T}$. von Kármán and J. Burgers, "General aerodynamic theory-perfect fluids," in Aerodynamic Theory (Julius Springer, Berlin, 1935).

${ }^{3}$ R. Knoller, "Die Gesetze des Luftwiderstandes," Flug- und Motortechnik 3, 1 (1909).

${ }^{4}$ A. Betz, "Ein Betrag zur Erklärung des Segelfluges," Zeitschrift für Flugtechnik und Motorluftshiffahrt 3, 269 (1912).

${ }^{5}$ I. Garrick, "Propulsion of a flapping and oscillating airfoil," NACA Technical Report 567, 1937.

${ }^{6}$ N. M. Koochesfahani, "Vortical patterns in the wake of an oscillating airfoil," AIAA J. 27, 1200 (1989).

${ }^{7}$ P. Freymuth, "Thrust generation by an airfoil in hover modes," Exp. Fluids 9, 17 (1990).

${ }^{8}$ G. S. Triantafyllou, M. S. Triantafyllou, and M. Grosenbaugh, "Optimal thrust development in oscillating foils with application to fish propulsion," J. Fluids Struct. 7, 205 (1993).

${ }^{9}$ J. Anderson, K. Streitlien, D. Barrett, and M. Triantafyllou, "Oscillating foils of high propulsive efficiency," J. Fluid Mech. 360, 41 (1998).

${ }^{10}$ M. Rosen, "Water flow about a swimming fish," Technical Report TP 2298 (US Naval Ordnance Test Station, China Lake, CA, 1959). 
${ }^{11}$ R. Blickhan, C. Krick, D. Zehren, and W. Nachtigall, "Generation of a vortex chain in the wake of a subundulatory swimmer," Naturwiss. 79, 220 (1992).

${ }^{12} \mathrm{~N}$. Vandenberghe, J. Zhang, and S. Childress, "A symmetry-breaking leads to forward flapping flight," J. Fluid Mech. 506, 147 (2004).

${ }^{13} \mathrm{~S}$. Childress and R. Dudley, "Transition from ciliary to flapping mode in a swimming mollusc: flapping flight as a bifurcation in $\operatorname{Re}_{\omega}$," J. Fluid Mech. 498, 257 (2004).

${ }^{14}$ J. Walker, "Functional morphology and virtual models: physical constraints on the design of oscillating wings, fins, legs, and feet at intermediate Reynolds numbers," Integr. Comp. Biol. 42, 232 (2002).

${ }^{15}$ S. Alben and M. Shelley, "Coherent locomotion as an attracting state for an oscillating free body," Proc. Natl. Acad. Sci. U.S.A. 102, 11163 (2005).

${ }^{16}$ E. Purcell, "Life at low Reynolds number," Am. J. Phys. 45, 3 (1977).

${ }^{17}$ S. Childress, Mechanics of Swimming and Flying (Cambridge University Press, Cambridge, UK, 1981).

${ }^{18}$ C. H. K. Williamson, "Vortex dynamics in the cylinder wake," Annu. Rev. Fluid Mech. 284, 477 (1996).

${ }^{19}$ J. Zhang, S. Childress, A. Libchaber, and M. Shelley, "Flexible filaments in a flowing soap film as a model for flags in a two dimensional wind," Nature (London) 408, 835 (2000)
${ }^{20}$ M. Shelley, N. Vandenberghe, and J. Zhang, "Heavy flags undergo spontaneous oscillations in flowing water," Phys. Rev. Lett. 94, 094302 (2005).

${ }^{21}$ V. Strouhal, "Uber eine besondere Art der Tonerregung," Ann. Phys. Chem. 5, 216 (1878).

${ }^{22}$ M. Triantafyllou, G. Triantafyllou, and D. Yue, "Hydrodynamics of fishlike swimming," Annu. Rev. Fluid Mech. 32, 33 (2000).

${ }^{23}$ G. Taylor, R. Nudds, and A. Thomas, "Flying and swimming animals cruise at a Strouhal number tuned for high power efficiency," Nature (London) 425, 707 (2003).

${ }^{24}$ K. D. Jones, C. M. Dohring, and M. F. Platzer, "Wake structures behind plunging airfoil: a comparison of numerical and experimental results," in 34th AIAA Aerospace Sciences Meeting, Reno, NV, 1996, AIAA Paper No. 96-0078.

${ }^{25} \mathrm{Z}$. J. Wang, "Vortex shedding and frequency selection in flapping flight," J. Fluid Mech. 410, 323 (2000).

${ }^{26}$ G. H. Keulegan and L. H. Carpenter, "Forces on cylinders and plates in an oscillating fluid," J. Res. Natl. Bur. Stand. 60, 423 (1958).

${ }^{27}$ R. P. Feynman, R. B. Leighton, and M. L. Sands, The Feynman Lectures on Physics (Addison-Wesley, Reading, MA, 1964), Vol. II, Chap. 16.

${ }^{28} \mathrm{P}$. Freymuth, "Propulsive vortical signature of plunging and pitching airfoils," AIAA J. 26, 881 (1988). 